\title{
Mango ripeness classification system using hybrid technique
}

\author{
Muhamad Farid Mavi ${ }^{1}$, Zulkifli Husin ${ }^{2}$, R. Badlishah Ahmad ${ }^{3}$, Yasmin Mohd Yacob ${ }^{4}$, \\ Rohani S. Mohamed Farook ${ }^{5}$, Wei Keong Tan ${ }^{6}$ \\ ${ }^{1,2,4,6}$ School of Computer and Communication Engineering, Universiti Malaysia Perlis, Malaysia \\ ${ }^{5}$ Faculty of Engineering Technology, Universiti Malaysia Perlis, Malaysia \\ ${ }^{3}$ Faculty of Informatics and Computing, Universiti Sultan Zainal Abidin, Malaysia
}

\begin{tabular}{l} 
Article Info \\
\hline Article history: \\
Received Sep 27, 2018 \\
Revised Nov 26, 2018 \\
Accepted Dec 6, 2018 \\
\hline Keywords: \\
Fruit ripeness \\
Hybrid / combination technique \\
Image processing \\
Odour sensing \\
SVM
\end{tabular}

Corresponding Author:

Dr. Zulkifli Bin Husin,

School of Computer and Communication Engineering,

Universiti Malaysia Perlis (UniMAP),

First Floor, Pauh Putra Campus, 02600 Arau, Perlis, Malaysia.

Email: zulhusin@unimap.edu.my

\begin{abstract}
Nowadays there are many systems develop for agricultural purposes and most system implemented on the use of non-destructive technique not only to classify but also to determine the fruit ripeness. However, most of the studies concentrates using single technique to assess the fruit ripeness. This paper presents the work on mango ripeness classification using hybrid technique. Hybrid stands for mix or combination between two different elements, thus this study combined two different technique that is image processing and odour sensing technique in a single system. Image processing technique are implemented using color image that is HSV image color method to determine the ripeness of fruit based on fruit peel skin through color changes upon ripening. Whereas, odour sensing technique are implemented using sensors array to determine the fruit ripeness through smell changes upon ripening. The "Harumanis" and "Sala" mango was used for sample collection based on two different harvesting condition that is unripe and ripe were evaluated using the image processing and followed by the odour sensor. Support Vector Machine (SVM) is applied as classifier for training and testing based on the data collected from both techniques. The finding shows around $94.69 \%$ correct classification using hybrid technique of image processing and odour sensing in a single system.
\end{abstract}

Copyright $\odot 2019$ Institute of Advanced Engineering and Science. All rights reserved.

\section{INTRODUCTION}

Fruits are consumed in Malaysia and globally whether it is eaten raw, cooked or consumed after being processed. It is because fruits guarantee health benefits such as energy supplier, disease prevention, healthy diets and well-built body in the long run. Fruits benefit the body immensely as they are natural sources of vitamins and minerals, which are essential for the proper functioning of the body, besides help to prevent from health complications such as cancer, high blood pressure, heat stroke, diabetes, etc. In Malaysia, fruits are divided either for fresh consumption usually for consumers and also production purposes that are develop by the industries [1]. Fresh consumption market where consumer will buy fresh fruits for daily intake or in cooking whereas production purposes market where the fruit are developed into edible purposes such as jams, sauces or marmalades but also for non-edible purposes, such as cosmetics, soaps, detergents, etc. The most important criteria when determine the fruits ripeness are the color of the skin, size and also odour where these three criteria are usually being classify by experts or farmers. However, the classification to determine the ripeness are often being evaluate differently by experts or farmers based on 
own individual understanding. Therefore, with the aid of image processing and odour sensing technique, the ripeness classification resulting more accurate, reducing error and eliminate the manual ways.

Digital image processing is vastly developed in many applications, thus its capabilities has attracted interest of several industries and chosen due to high consistency with accuracy [2]. Over recent years, this technology is ideal in the agricultural sector, where it can be used for quality assessment and classification based on size or maturity level of fruits [3]. Furthermore, using image processing can replaced the traditional technique that used to evaluate the fruit since ages because it uses non-destructive evaluation method. Odour sensing or odour gas sensor are also been recognized as hardware implementation for fruit ripeness classification. It is a fact that the smell or aroma can recognized the flavour, odour and volatile compounds inside the fruit. The odour sensor able to measure consistently because it can measure and identify quality factors such as impurities, taints and adulteration [4]. Besides that, odour sensor also have advantages that includes rapid, real-time volatiles detection and cheaper costs [4]. Therefore, this paper explores the implementation of image processing and odour sensing method together in a single system as a hybrid technique for mango ripeness classification.

Based on the previous literature review by other researches, in [5], a study conducted to predict quality of "Alphonso" mangos using RGB images. Hierarchical clustering method was used to classify ripening period based on physico-chemical, color and textural parameters. Normalized differential index and area features models resulted $96.3 \%$ of correct prediction that are extracted from the images. Besides that, [6] proposed an approach using mean pixel values in RGB and HSB color spaces. The "Chokanan" mango was selected and a model was develop based on hue from the HSB using linear regression analysis with average success rate of $95.67 \%$. Authors [7] implemented system that detect "Gincu" mango ripening through color using $\mathrm{C}$ language, computer vision and artificial neural network. The mango fruit sortation classifies with $94 \%$ of accuracy and also conclude that speed and accuracy are important when handling with hidden layer using neural network.

On the other hand, there are also previous research that implemented odour sensing towards mango fruit for ripeness classification. [4] implemented an array of semi-conductor gas sensors to determine the pattern of "Harumanis" mango ripeness. Each stages of ripeness are identified and trained using artificial neural network where this method proven that the sensor array able to leaves a characteristic pattern for different stages of ripeness. Authors [8] also using semi-conductors gas sensors using Principal Component Analysis (PCA) and also Artificial Neural Network (ANN) for ripeness assessment for "Harumanis" mango. It also concluded that the particular method able to leaves significant characteristic pattern through different ripeness level. In [9] can also determine the "Chokanan" mango ripeness using zNoseTM electronic nose that used electronic detector with basis of (gas) chromatography with system controller. The system potentially able to predict mango fruit maturity during harvesting at the optimal harvest time. Therefore, based on the previous studies, color and smell are important parameter to identify the mango ripeness, thus by implementing image processing and odour sensing together as an automated system increases the reliability and accuracy for mango ripeness classification.

\section{RESEARCH METHOD}

\subsection{Image Processing Technique \\ 2.1.1 Fruit Preparation}

Mango images are used for fruit ripeness based on the fruit peel color changes. "Harumanis" and "Sala" mangos are selected as it differs in term of color changing upon ripening. The mangos skin differs from unripe to ripe, for example each level of ripeness based on the mangos skin sometimes can be distinguished by naked eye. Unripe can be determine when the skin is greenish and will completely or slightly changing to yellowish color upon ripening. Sometimes there are also mangos that change from darker green to lighter green when it is ripe but there also no color changes upon ripening for some mangos but other properties enable to identify its ripeness such as odour. Figure 1 illustrates the "Harumanis" and "Sala" mango images that has different tone color skin correlations to identify whether it is unripe or ripen.

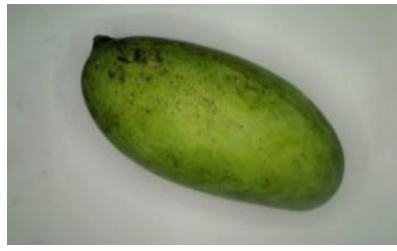

(a)

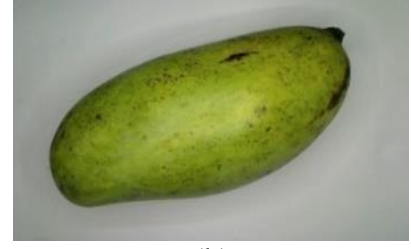

(b) 


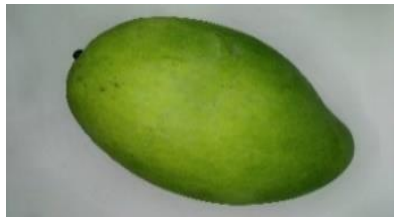

(c)

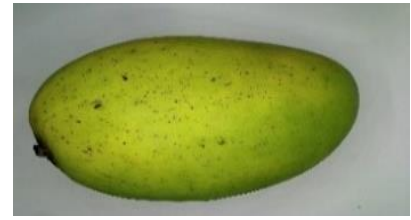

(d)

Figure 1. Different type of mangos where (a) and (b) is the "Sala" mango whereas (c) and $(\mathrm{d})$ is the "Harumanis" mango

\subsubsection{Sample Collection}

The data set of "Harumanis" and "Sala" mango samples with total of 228 images each respectively used for image processing. For image processing technique, these fruit images are divided into training and testing set, where $30 \%$ of the fruit images from each group are used to train the technique and remaining images serve as the testing set. The fruit samples are manually harvested from Bukit Temiang, Perlis to ensure randomness in fruit collection process. The samples are also being proved by the experts in the relevant field for manual prediction of maturity so that it can be compared to the techniques that are used in the system. The fruit data are captured using Logitech720p Webcam C905 shown in Figure 2 and saved at the database consists of all the images that are separated by different folder according to its categories so that it is easy to retrieve.

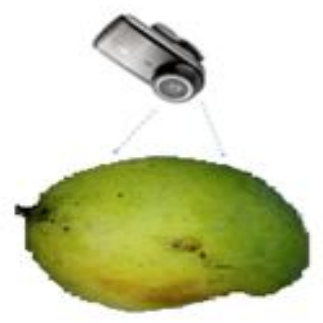

Figure 2. Fruit captured for data acquisition using Logitech720p Webcam C905

\subsubsection{Pre-Processing}

Pre-processing is a useful and important step in order to increase overall accuracy of image processing technique and the target image should same attribute. It is also a technique used for eliminating insignificant information based on data collection of certain pixels and therefore acquiring the images are important to ensure all variable are controlled to make sure that the data are valid. In pre-processing, it is necessary that all images being are in similar category so that the images can processed accurately. The images undergone image pre-processing and for this case, the images are pre-processed when the photo are taken by using white background when the image are captured. White background is chosen as the fruit background as the controlled parameter because it can capture image clearer and eliminate excess lighting from the surroundings.

\subsubsection{RGB to HSV Conversion}

The sample collected are in form of the RGB spectrum that is in red, green and blue channel with each pixel in the channels having value ranging from 0 to 255 [3]. After that, the RGB will be convert into HSV color model where the image is separated into hue, saturation and value channel or image using set of equations from (1), (2), (3), (4), (5). In HSV, the value varies between 0 and 1 for each channel and Figure 3 illustrate the difference when an original image converted to hue, saturation and value channel. The conversion from RGB to HSV is to detect the color presence in the images more accurately based on image acquired. The significant advantages of HSV than other color spaces is the compatibility that is similar to human intuition besides the possibility of using single, two or all color feature that allowing the implementation of much faster algorithms and also accurate result.

$$
H_{1}=\cos ^{-1}\left\{\frac{\frac{1}{2}[(R-G)+(R-B)]}{\sqrt{(R-G)^{2}+(R-B)(G-B)}}\right\}
$$




$$
\begin{aligned}
& H=H_{1}, \text { if } B \leq G \\
& H=360^{\circ}-H_{1}, \text { if } B>G \\
& S=\frac{\max (R, G, B)-\min (R, G, B)}{\max (R, G, B)} \\
& V=\frac{\max (R, G, B)}{255}
\end{aligned}
$$

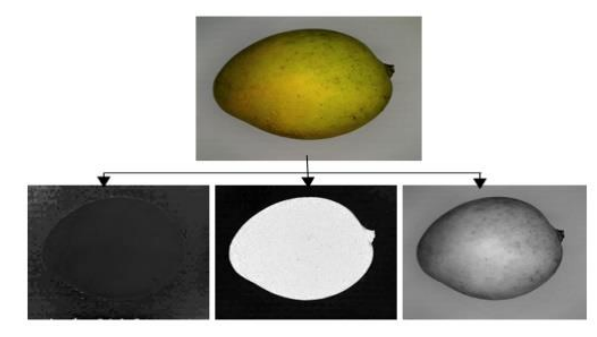

Figure 3. Original image (RGB) converted to HSV color model

Fruits can be categorized into several ripeness or maturity levels based on fruit skin color changes. For this implementation of fruit using HSV, mango images are used to test the accuracy and reliability of the methods. The fruit skin differs from unripe to ripe, for example each level of ripeness can be set apart based on the fruit skin color. Unripe can be determine when the skin is pale according to the fruit and will slightly or completely changed to another color upon ripening. Therefore, the HSV color space or model correspond closely to color perception that similar to human interpretation [3].

\subsubsection{Thresholding}

In this stage, there are used of threshold method in order to isolate the colors by applying multiple masks. A low threshold and high threshold mask are implemented for hue, saturation and value images. Anything pixel within these thresholds will be set to 1 and the remaining pixels will be zero. In threshold method, the global threshold (level) played an important role in order to set a boundary of HSV grayscale value. In this concept, graythresh function in MATLAB which is represented as global image threshold using Otsu's method is applied to compute the threshold level, which can minimize the intraclass variance of the black and white pixel [10]. By using the fuction, the threshold masking levels are obtained and produced as binary output.

\subsubsection{Classification}

The data images that have been processed are analyzed to determine the fruit ripeness. Each pixel of image containing RGB and HSV color space data and each color component ranging of 0 to 255 for RGB and range of 0 to 1 for HSV. The system applied Support Vector Machine (SVM) learning machine for ripeness stages classification based on the image processing technique data. The inputs are the training dataset feature vectors that is the mean HSV value from the HSV conversion and their corresponding classes, whereas the outputs are the ripeness stage of the image in the testing dataset. SVM first transform the input data into a higher-dimensional space by using a kernel function and an optimal hyperplane is constructed between two classes whether it is unripe or ripe to separate the data in transformed space [11]. Specific range feature decides on to acquire specific result for example if the mean HSV is less or higher than the boundary that have been set, thus it can determine whether the mango is unripe or ripe. The inputs value trained using the SVM classifier and later test the accuracy.

\subsection{Odour Sensing Technique \\ 2.2.1 Odour Data Acquisition}

The mango fruits are also applied in odour sensing technique for ripeness classification. This odour sensing technique are not similar with image processing technique. Odour sensors can sense and record the fruit smell whether it is ripe or unripe because different fruit conditions or maturity gives different reading. There are several types of metal oxide gas sensors implemented and applied in this hardware development as stated in Table 1 [12]. 


\begin{tabular}{|c|c|c|}
\hline Label & Sensors & Odour Types \\
\hline S1 & TGS813 & Combustible Gases \\
\hline S2 & TGS821 & Hydrogen \\
\hline S3 & TGS822 & Ethanol \\
\hline S4 & ME3-C2H4 & Ethylene \\
\hline S5 & TGS825 & Hydrogen Sulphide \\
\hline S6 & TGS826 & Ammonia \\
\hline S7 & TGS830 & Chlorofluorocarbons \\
\hline S8 & TGS2600 & Air Contaminants \\
\hline S9 & TGS2620 & Alcohol \& Solvent Vapours \\
\hline
\end{tabular}

The hardware development which consists of the sensor arrays or Odour Gas Sensor (OGS) with addition of exhausted fan, temperature and humidity display are implemented together in the prototype. The initial sensor arrays prototype started with circuit design of sensors and before tested on the PCB board. The circuit can convert the changing in conductivity to output signal with corresponding to gas concentration. Based on the characteristics of the metal gas oxide, where the changing of resistance and voltage of sensors happen when it reacts between the negative charge of metal oxide surface and deoxidizing gas. The odour data based on the mangos sample reading was taken to differentiate the level of maturity from each batch of fruits one by one. The odour data from the OGS was obtained through the National Instrument (NI) Data Acquisition (DAQ) device, where it can read the volatilities changes from the sensor. The NI-DAQ device also recorded the odour data as a reference library for training and testing. Therefore, by using OGS it can differentiate the mango ripeness through smell properties and by implementing several gas sensors also will also increase the accuracy of fruit smell and aroma detection and also classification [12].

\subsubsection{Odour Pre-Processing}

As for odour pre-processing it is slightly different than image pre-processing as the odour data must also be valid where all the variable has been controlled. There are issues when using metal gas oxide, which it had a high sensitivity to temperature and humidity. Therefore, the developed OGS are installed in the prototype together with the webcam camera that appear similar to a gas chamber box for data collection as shown in Figure 4. Two exhausted fans are also fixed at the prototype to ensure that after the odour reading collection, the excess odour inside the prototype are eliminate using the fan. Thus, the prototype surrounding is at initial state and the data collection for other samples will be valid and accurate. OGS will detect the mango odour, thus the output voltage from the sensor will be sent to NI-DAQ for data collection, processing and classification through the computer.

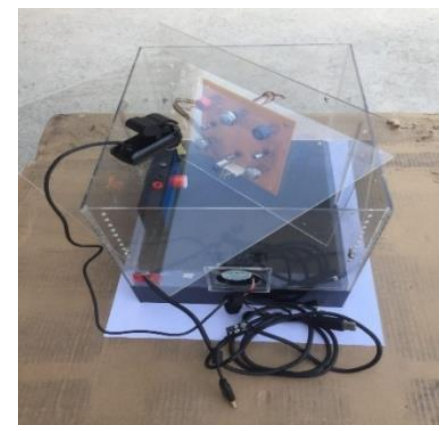

Figure 4. Prototype that implemented image processing and OGS in a single system

\subsubsection{Odour Measurement Technique}

Measurement process for odour sensing technique are divided into three different part or phases that is initialization, measuring, and purging. The measurement process starts by placing mangos in the prototype chamber for initializes phase. To strengthen the aroma or smell concentration and obtaining higher sensor responses, the sensors boot up to a steady state after 200s. After that, the measurement phase starts where sensors detected the mango smell and reading through NI-DAQ device for around 300s, which enough for sensors obtained stable value. Lastly the purging phase will occur after the measurement complete. The main purpose of this phase is to remove the excess odour by using surrounding air through the fan so that the sensors could go to the initial baseline values [13]. After the excess odour existed in the prototype chamber is 
exhausted by the fan, new measurement and reading started again. The collected reading was conducted at the temperature around $27^{\circ} \mathrm{C}$ to $33^{\circ} \mathrm{C}$ and maintained constant. The data value was collected from the odour gas sensor (OGS) using the NI DAQ device and NI LABVIEW software is used to display the waveform chart consisting of each sensor data through the Graphical User Interface (GUI) inside NI LABVIEW software.

\subsubsection{Classification}

SVM learning machine as classifier are also used for odour sensing training and testing, where it is useful to understand which combinations of pairs of sensors are gives the able to differentiate between ripe and unripe smell. By testing the SVM with untrained data, the classifier is able to detect the maturity level of mangos using OGS technique. System started and test continuously and the mean value are calculated for each sensor output to feed into the SVM for training. Using SVM, the method performs without waiting for the sensor to fully reach up to a constant value whereby a quick detection method of fruit maturity is detected in a few seconds based on the rising signal of the odour sensor.

\subsection{Image and Odour Combination (Hybrid Technique)}

As mentioned earlier, hybrid stands for combination between two different elements and this study implemented software and hardware combination by using image processing technique and also OGS technique. The approach is to put the both techniques side-by-side and focus on their interaction in a problem-solving task. The conceptual view allows one to abstract from the individual techniques as well as to study the individual contribution of each component. The benefits of integrated techniques include robustness, improved performance and increased problem-solving capabilities for classification [14]. Figure 5 illustrated the image processing and OGS using hybrid technique for fruit ripeness classification. Hybrid systems have limitations caused by the increased complexity therefore, both of the technique is integrated together in LabVIEW software that also acts as GUI for simpler solution. The image processing process and also SVM classifier that are employed through MATLAB software are integrated with the OGS hardware, thus the LabVIEW acts as the integrated medium for the fruit ripeness classification decision in a single system.

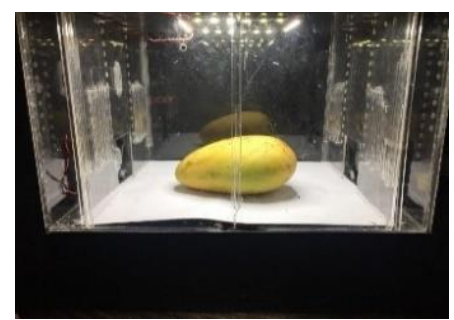

(a)

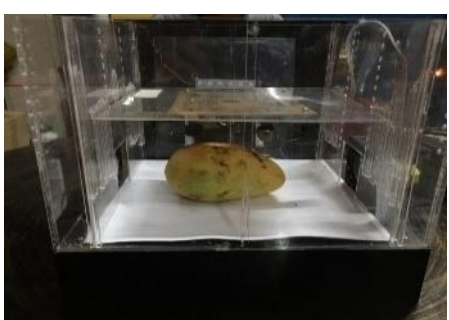

(b)

Figure 5. Prototype that implemented image processing (a) and OGS (b) in a single system

\section{RESULTS AND ANALYSIS}

After the fruit data has been collected and processed through image processing along with odour sensing, the data are separated whereby $30 \%$ used for training and the remaining used for testing. The reason using this ratio is because to decrease the time for samples training and increase the accuracy result through increment of the testing samples. Table 2 tabulated the samples used for training and testing with two different classes that is unripe and also ripe, besides the SVM learning machine are used for training and testing so that the system can classify the mango ripeness.

Table 2. Samples Used for Training and Testing

\begin{tabular}{lccccc}
\hline \multirow{2}{*}{ Fruits } & \multirow{2}{*}{ Total Fruit Samples } & \multicolumn{3}{c}{ No. of Fruit Samples Used for } \\
& & Training & \multicolumn{2}{c}{ Testing } \\
& 228 & 34 & 34 & 80 & 80 \\
\hline Harumanis mango & 228 & 34 & 34 & 80 & 80 \\
Sala mango & 456 & 68 & 68 & 160 & 160 \\
Total & & & Rnipe & Unripe & Ripe \\
\hline
\end{tabular}


To ensure that the system are operating and robust, the accuracy of the system for mango ripeness classification are calculated using (6).

$$
\text { Accuracy }=\frac{T P+T N}{T P+F N+F P+T N}
$$

Where $: T P=$ true positive

$T N=$ true negative

$F P=$ false positive

$F N=$ false negative

Figure 6 shows the mean HSV value corresponding to the mango images according to the respective levels of ripeness. Based on the result, there are difference for unripe and ripe level can be seen in each $\mathrm{H}$, $\mathrm{S}$ and $\mathrm{V}$ value. The mean $\mathrm{H} \& \mathrm{~V}$ differs slightly around its value area whereas, mean $\mathrm{V}$ plays the crucial value to determine the mean HSV and also the ripeness condition where the unripe level are around 0.6 and below whereas ripe level delivers 0.6 and above. The graph also shows that the hue reading linearly increase when the the saturation and value channel reading decrease based on the images running in the program.

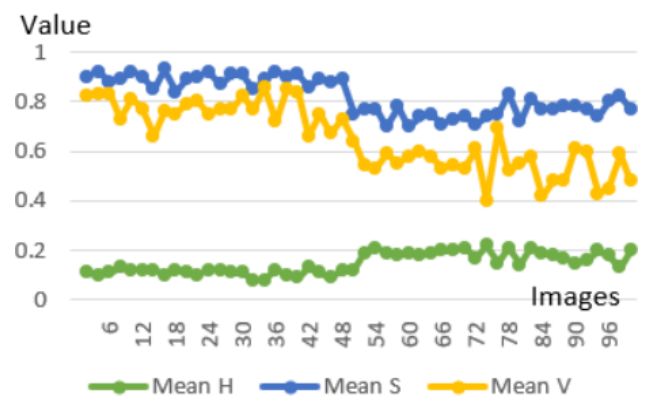

Figure 6. Mean value for ripeness level using HSV

The result from Figure 7 shows the histogram data for the test image classification through the SVM technique. Based on the histogram, the hue value is not entirely differing for unripe or ripe images, but for the saturation and value it is not similar. It can be seen that the saturation peak for the ripe fuit is higher than the unripe fruit. Whereas for the value histogram, the ripe fruit peak value is near to the threshold, while for the unripe fruit the peak is slightly further away from the threshold value that is 0.6. Therefore, the HSV image color basically with addition of thresholding and SVM as classifier can determine the ripeness for mango fruit images and theoretically it correspond similar to the color intepretation by human.
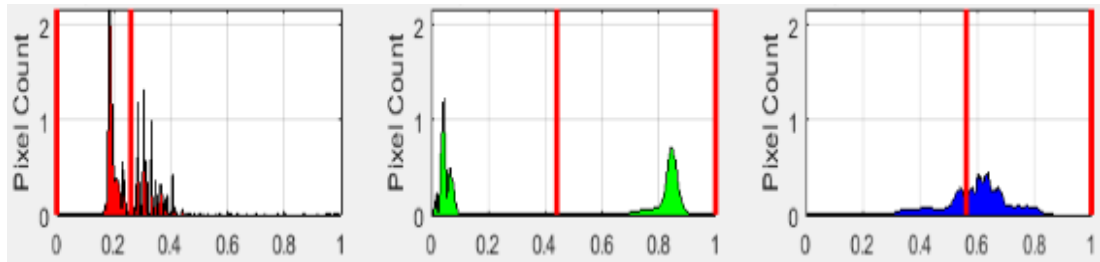

(a)
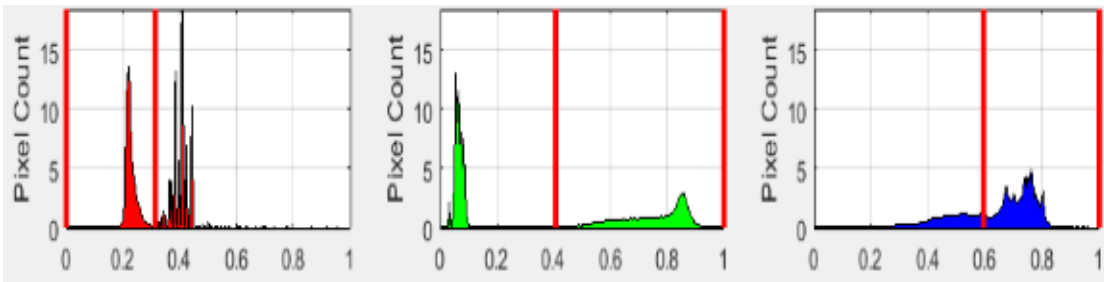

(b)

Hue

Saturation

Value

Figure 7. Histogram analysis graph for (a) unripe fruit (b) ripe fruit 
Table 3 summarizes the classification result using the image processing technique proved that the technique able to achieve high precision of $91.88 \%$ of accuracy in determine fruit ripeness. Based on the result, it can classify and determine all of the 456 images of mangos. There are some misclassify samples due to some mango images that contained more greenish color when it ripe and also some yellowish color when it is actually still unripe. Nevertheless, the overall performance by using image processing to determine the fruit classification is good and the technique is suitable for certain fruit that changed color from unripe to ripe such as mango

Table 3. Classification of Mango Images using Image Processing Technique

\begin{tabular}{lccccc} 
& \multicolumn{5}{c}{ For Image } \\
& TP & FN & TN & FP & Acc \\
\hline Harumanis mango & 76 & 4 & 74 & 6 & 93.75 \\
Sala mango & 74 & 6 & 70 & 10 & 90.00 \\
Total & 150 & 10 & 144 & 16 & 91.88 \\
\hline
\end{tabular}

The sensors that indicates the difference of fruit ripeness are shown in Figure 8 where the chart illustrates the sensor reading between unripe and ripe. Pairs of sensors which made the best discrimination between unripe and ripe class are sensors S3 (ethanol) and S4 (ethylene) that provided the huge difference reading of sensors than other sensors for discriminating the ripening stages.

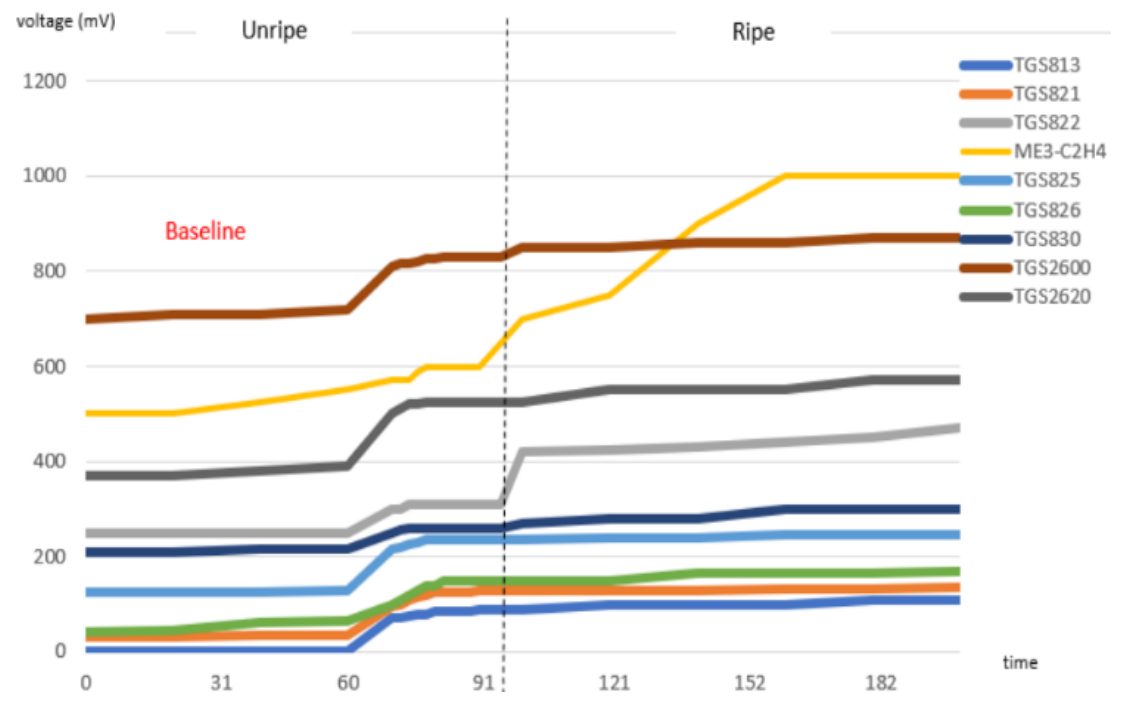

Figure 8. Sensor array reading using OGS for unripe and ripe mangos

Table 4 sum up the classification result using odour sensing technique which shows the odour gas sensor array able to achieve high precision of $97.50 \%$ of accuracy in determine fruit ripeness. Similar amount of data that is 456 used to match the images data and hence, it can classify and determine all of the 456 odour data of mangos with few are misclassify maybe because some reading recorded stronger smell although it is still unripe. The overall performance by using odour sensing to determine the fruit classification is suitable and technique is fit for fruit that that has high concentration of smell or aroma from unripe to ripe stage that are similar as mango.

Table 4. Classification of Mango Images using Odour Sensing Technique

\begin{tabular}{lccccc}
\hline & \multicolumn{5}{c}{ For Odour } \\
& Unripe & Ripe & Acc \\
& TP & FN & TN & FP & \\
Harumanis mango & 78 & 2 & 80 & 0 & 98.75 \\
Sala mango & 74 & 6 & 80 & 0 & 96.25 \\
Total & 152 & 8 & 160 & 0 & 97.50 \\
\hline
\end{tabular}


As a result, the overall accuracy of fruit ripeness classification through image processing and odour sensing as a hybrid system achieved accuracy of $94.69 \%$ by combining both accuracy that is $91.88 \%$ and also $97.50 \%$. The main point is that the hybrid technique based on image processing and odour sensing can accommodate each other. This means that if the mango ripeness cannot identify accurately using image processing, it can be identified through odour sensing and vice versa. Hence, the system can be implemented for other fruits because has the ability to cover wider range of fruits that have either color or smell properties upon ripening.

\section{CONCLUSION}

In this paper, hybrid technique as a combination method used for mango ripeness classification by implementing image processing and odour sensing technique. The hybrid approach is to put the both techniques side-by-side and can accommodate each other in case the fruit cannot be determine using single technique. HSV color models and array gas sensors used as basic development to extract the required information to classify mango ripeness. The data obtained for the classification shows that both the unripe group and ripe group can be achieved and identified with an accuracy of $94.69 \%$. As a conclusion, the image processing technique combined with odour sensing able to differentiate ripeness in mango and using the hybrid technique it can be implement for other types of fruits in the future.

\section{ACKNOWLEDGEMENTS}

The author would like to acknowledge the support from the Fundamental Research Grant Scheme (FRGS) under a grant number of FRGS/1/2015/ICT02/UNIMAP/03/1 from the Ministry of Higher Education Malaysia.

\section{REFERENCES}

[1] M. Ronald and M. Evans, "Classification of Selected Apple Fruit Varieties using Naive Bayes," Indian Journal of Computer Science and Engineering, pp. 13-19, 2016.

[2] R. Gonzalez and R. Woods, Digital Image Processing, 3rd ed., Peason Prentice Hall, 2008.

[3] K. Plataniotis and A. Venetsanopoulos, Color Image Processing and Applications, Springer-Verlag, 2000, pp. 1-65.

[4] S. N. M. Salim, A. Y. M. Shakaff, M. N. Ahmad and A. H. Adom, "A Feasibility Study of Using an Electronic Nose as a Fruit Ripeness Measuring Instrument," in 1st International Workshop on Artificial Life and Robotics, 2005.

[5] V. E. Nambi, K. Thangavel and V. Thirupathi, "Comparison of Various RGB Image Features for Nondestructive Prediction of Ripening Quality of "ALPHONSO" Mangoes for easy Adoptability in Machine Vision Applications: A Multivariate Approach," Journal of Food Quality, pp. 816-825, 2016.

[6] S. K. Bejo and S. Kamaruddin, "Determination of Chokanan mango sweetness (Mangifera indica) using nondestructive image processing technique," Australian Journal of Crop Science, pp. 475-480, 2014.

[7] E. H. Yossy, J. Pranata, T. Wijaya, H. Hermawan and W. Budiharto, "Mango Fruit Sortation System using Neural Network and Computer Vision," in 2nd International Conference on Computer Science and Computational Intelligence, Bali, 2017

[8] S. N. M. Salim, A. Y. M. Shakaff, M. N. Ahmad, A. H. Adom and Z. Husin, "Development of electronic nose for fruits ripeness," in International Conference on Sensing Technology, Palmerston North, 2005.

[9] F. G. Nouri, Z. Chen and M. Maqbool, "Monitoring Mango Fruit Ripening after Harvest using Electronic Nose (zNoseTM) Technique," in International Conference on Food Engineering and Biotechnology, Singapore, 2014.

[10] O. Nobuyuki, "A Threshold Selection Method from Gray-Level Histogram," in IEEE Transaction on Systems, Man \& Cybernatics, 1977.

[11] R. Ruiz-Gonzalez, J. Gomez-Gil, F. J. Gomez-Gil and V. Martinez-Martinez, "An SVM-Based Classifier for Estimating the State of Various Rotating Components in Agro-Industrial Machinery with a Vibration Signal Acquired from a Single Point on the Machine Chassis," Sensors, pp. 20713-20735, 2014.

[12] M. M. Rahman, C. Charoenlarpnopparut, P. Suksompong and P. Toochinda, "Sensor Array Optimization for Complexity Reduction in Electronic Nose System," Transactions on Electrical Eng., Electronics and Communications, pp. 49-59, 2017.

[13] R. S. A. Karim, S. S. Abdul and M. A. Mikdad, "Post-harvest Quality Evaluation of Grapes using Non-destructive Electronic Nose," Journal of Electrical and Electronics Engineering, pp. 35-38, 2015.

[14] C. Grosan and A. Abraham, Hybrid Intelligent System, Springer-Verlag Berlin Heidelberg, 2011. 


\section{BIOGRAPHIES OF AUTHORS}

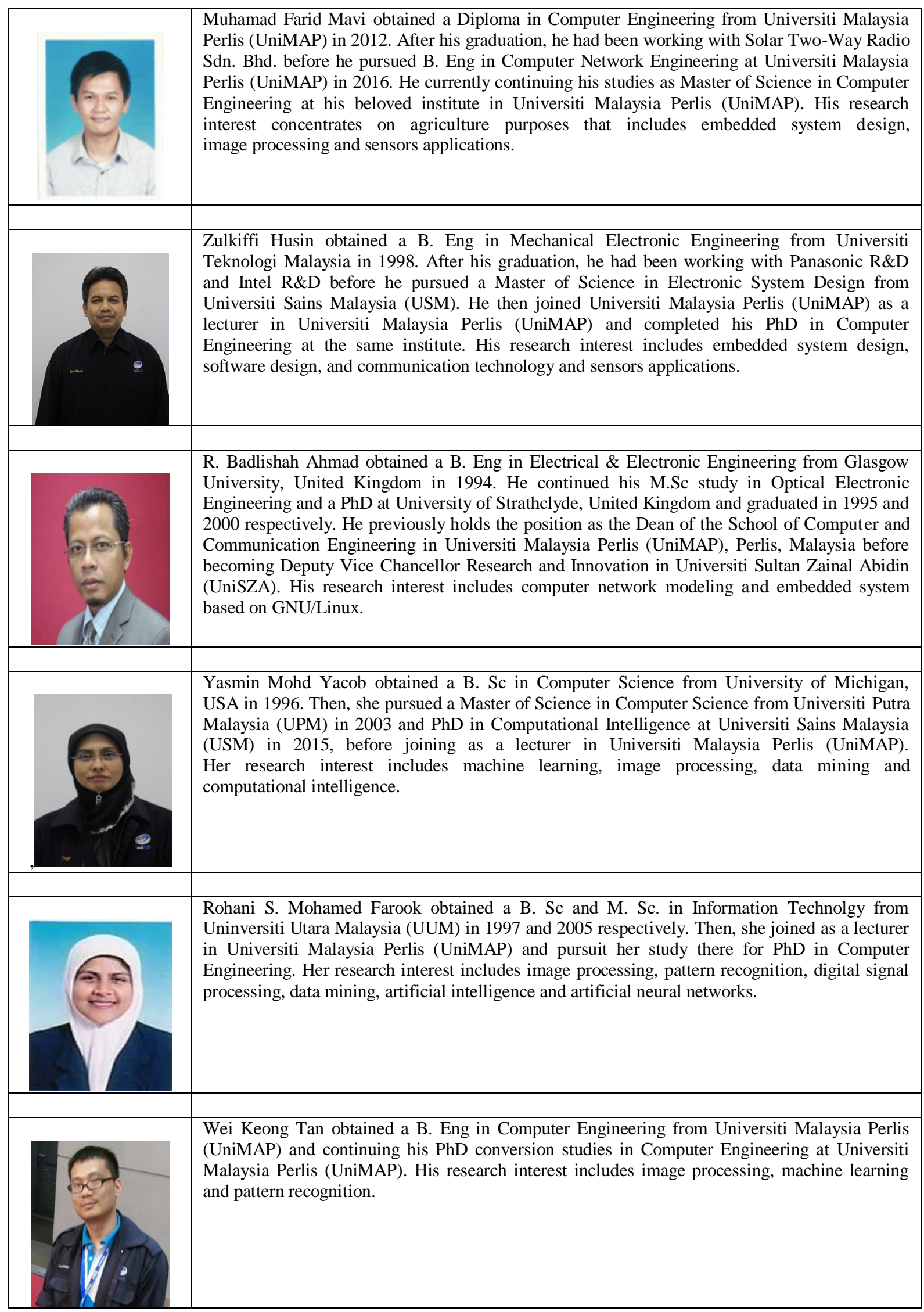

